\title{
Divulgação de informações sobre higiene e mudança de hábitos durante a pandemia
}

\section{da Covid-19}

\author{
Disclosure of information on hygiene and changing habits during the Covid-19 pandemic \\ Divulgación de información sobre higiene y cambio de hábitos durante la pandemia Covid-19
}

Recebido: 07/01/2021 | Revisado: 08/01/2021 | Aceito: 13/01/2021 | Publicado: 17/01/2021

Noádia Priscila Araújo Rodrigues

ORCID: https://orcid.org/0000-0003-2721-5768

Universidade Federal da Paraíba, Brasil

E-mail: noadia_priscila@ hotmail.com

Daniella Rocha da Silva

ORCID: https://orcid.org/0000-0002-5164-8718

Universidade Federal da Paraíba, Brasil

E-mail: daniellarocha94@gmail.com

Élcio Antônio Garcia Júnior

ORCID: https://orcid.org/0000-0001-8301-8190

Universidade Federal da Paraíba, Brasil

E-mail: elgarciajr@gmail.com

Edmilson Fernandes da Silva Júnior

ORCID: https://orcid.org/0000-0001-7157-1773 Universidade Federal da Paraíba, Brasil

E-mail: profedfernandes1998@gmail.com

Renally da Silva Gomes

ORCID: https://orcid.org/0000-0002-9726-2984 Universidade Federal da Paraíba, Brasil E-mail: renallygomes14@gmail.com

Karina Felix Dias Fernandes

ORCID: https://orcid.org/0000-0002-9867-3814 Universidade Federal da Paraíba, Brasil

E-mail: kfdfernandes@gmail.com

Kataryne Árabe Rimá de Oliveira

ORCID: https://orcid.org/0000-0002-4582-333X

Universidade Federal da Paraíba, Brasil

E-mail: katarynearabe_@hotmail.com

\begin{abstract}
Resumo
Covid-19 é uma doença respiratória aguda, causada pelo vírus SARS-CoV-2, que foi categorizada como pandemia em 2020. Sua contaminação acontece através de gotículas do vírus ativo, que pode ser transmitido entre indivíduos ou através de objetos contaminados. Mediante esses aspectos o presente trabalho teve como objetivo verificar se a busca por informações sobre medidas de higiene promoveu mudanças de hábitos nas práticas de higiene durante a pandemia da Covid-19. Para isso foi realizado um estudo transversal, através de questionário on-line (na plataforma Google formulários), que continha perguntas relacionadas ao período de emergência sanitária. A análise dos dados foi realizada através de estatística descritiva e inferencial por meio do teste de Qui-Quadrado de Pearson. Dentre os respondentes, a maioria declarou buscar informações sobre higiene para mitigar a contaminação do coronavírus durante o período da pandemia, com destaque para informações específicas sobre higiene de alimentos. As estratégias mais utilizadas para obter informação sobre práticas de higiene foram: internet, telejornais e e-book sobre boas práticas de prevenção ao Coronavírus. Dentre essas a internet influenciou positivamente nos hábitos de higiene de alimentos e uso correto de máscara. Ainda foi observado que os participantes mudaram os hábitos de aquisição de alimentos no que diz respeito a observar o prazo de validade e higienizar as embalagens no domicilio. É possível concluir que o período de pandemia gerou mudanças importantes nos hábitos de higiene da população estudada, e que estratégias de educação sanitária podem gerar transformações positivas nas práticas de produção de alimentos seguros.
\end{abstract}

Palavras-chave: Coronavírus; Covid-19; Vigilância sanitária; Hábitos de higiene; Segurança de alimentos.

\section{Abstract}

Covid-19 is an acute respiratory disease, caused by the SARS-CoV-2 virus, which was categorized as a pandemic in 2020. Its contamination occurs through droplets of the active virus, which can be transmitted between individuals or through contaminated objects. Based on these aspects, the present study aimed to verify whether the search for information on hygiene measures promoted changes in habits in hygiene practices during the Covid-19 pandemic. For 
this, a cross-sectional study was carried out through an online questionnaire (on the Google forms platform), which contained questions related to the health emergency period. Data analysis was performed using descriptive and inferential statistics using Pearson's chi-square test. Among the respondents, the majority declared to seek information on hygiene to mitigate the contamination of the coronavirus during the pandemic period, with emphasis on specific information on food hygiene. The most used strategies to obtain information on hygiene practices were: internet, news, and e-book on good Coronavirus prevention practices. Among these, the internet had a positive influence on food hygiene habits and the correct use of a mask. It was also observed that the participants changed the habits of purchasing food with regard to observing the expiration date and cleaning the packaging at home. It is possible to conclude that the pandemic period generated important changes in the hygiene habits of the population studied, and that health education strategies can generate positive changes in the practices of producing safe food.

Keywords: Coronavirus; Covid-19; Health Surveillance; Hygiene habits; Food safety.

\section{Resumen}

Covid-19 es una enfermedad respiratoria aguda, causada por el virus SARS-CoV-2, que fue catalogado como pandemia en 2020. Su contaminación ocurre a través de gotitas del virus activo, que puede transmitirse entre individuos o mediante objetos contaminados. A partir de estos aspectos, el presente estudio tuvo como objetivo verificar si la búsqueda de información sobre medidas de higiene promovió cambios en los hábitos en las prácticas de higiene durante la pandemia Covid-19. Para ello, se realizó un estudio transversal a través de un cuestionario online (en la plataforma de formularios de Google), que contenía preguntas relacionadas con el período de emergencia sanitaria. El análisis de los datos se realizó mediante estadística descriptiva e inferencial mediante la prueba de chi-cuadrado de Pearson. Entre los encuestados, la mayoría declaró buscar información sobre higiene para mitigar la contaminación del coronavirus durante el período pandémico, con énfasis en información específica sobre higiene alimentaria. Las estrategias más utilizadas para obtener información sobre prácticas de higiene fueron: internet, noticias y libro electrónico sobre buenas prácticas de prevención del coronavirus. Entre ellos, Internet influyó positivamente en los hábitos de higiene alimentaria y el uso correcto de una mascarilla. También se observó que los participantes cambiaron los hábitos de compra de alimentos con respecto a observar la fecha de vencimiento y limpiar el empaque en casa. Es posible concluir que el período pandémico generó cambios importantes en los hábitos de higiene de la población estudiada, y que las estrategias de educación en salud pueden generar cambios positivos en las prácticas de producción de alimentos inocuos.

Palabras clave: Coronavirus; Covid-19; Vigilancia sanitaria; Hábitos de higiene; Seguridad alimentícia.

\section{Introdução}

Covid-19 é uma doença respiratória aguda que foi categorizada como pandemia no ano de 2020. É causada pelo vírus SARS-CoV-2, pertencente à família dos coronavírus, um grande grupo de vírus que podem causar doenças respiratórias, gastrointestinais, hepáticas e neurológicas (Wu et al., 2020; WHO, 2020).

Sua transmissão pode ocorrer através de gotículas de saliva expelidas, principalmente, ao tossir, falar ou espirrar (Morawska \& Milton, 2019; WHO, 2020). Dessa forma, a contaminação acontece ao respirar estas gotículas contendo o vírus transmissor ativo, que pode ser transmitido de indivíduo para indivíduo ou por meio de objetos contaminados (WHO, 2020; Li et al, 2020). Estudos revelam que o vírus pode permanecer ativo em superfícies inanimadas por até 96 horas (Teixeira \& de Carvalho, 2020).

Dentre as estratégias de mitigação da doença, a principal tem sido o distanciamento social, que envolve restringir encontros sociais, minimizar aparições públicas e promover o trabalho em residência, conhecido como "home office". O uso de máscaras e a lavagem frequente de mãos também são estratégias adotadas para esta finalidade, que se baseiam na alta capacidade de transmissão do SARS-CoV-2 por vias aéreas e superfícies de contato (WHO, 2020; Morawska \& Milton, 2020; Villapol, 2020).

Outro fator importante para a alta taxa de transmissão da doença é o fato de que, ao contrário de outros vírus da família dos coronavírus, o SARS-CoV-2 pode ser disseminado por pessoas que apresentam poucos ou nenhum dos sintomas (Morawska \& Milton, 2020; Villapol, 2020).

As medidas de mitigação para Covid-19, no Brasil, iniciaram em 28 de fevereiro de 2020, no distrito federal, e em seguida, os demais estados da federação aderiram aos protocolos de segurança recomendados pelos órgãos internacionais de saúde (Rodriguez-Morales et al, 2020; Ministério da Saúde, 2020). 
Postula-se que houve maior busca por informações relacionadas às práticas de higiene para mitigar a propagação da COVID-19, sobretudo em fontes de informações relacionadas à higiene de alimentos, pois, com os estabelecimentos fechados, tais como bares e restaurantes, houve maior demanda para aquisição de alimentos prontos ou insumos para fabricação de alimentos em ambiente doméstico (Romeo-Arroyo et al, 2020).

Mediante o exposto, este estudo foi desenvolvido com o objetivo de verificar se a busca por informações sobre medidas de higiene promoveu mudanças de hábitos nas práticas de higiene durante a pandemia da Covid-19.

\section{Metodologia}

O delineamento de estudo do presente trabalho caracteriza-se como uma pesquisa exploratória, transversal de natureza quali-quantitativa (Pereira et al., 2018), direcionado a pessoas maiores de 18 anos com acesso a redes sociais, respeitando as normas das resoluções CNS/MS 466/12 e 510/16 (Ministério da Saúde, 2013; Ministério da Saúde, 2016), por meio de um questionário on-line contendo quatro perguntas de cunho sociodemográfico, três perguntas a respeito da busca de informações sobre higiene de alimentos, quatro perguntas acerca das mudanças nas práticas de higiene pessoal e onze perguntas sobre as mudanças nas práticas de higiene de alimentos. Todas as questões foram relacionadas ao período de emergência sanitária devido ao Covid-19. Os questionários foram criados e hospedados no site da plataforma Google formulários.

A coleta de dados foi realizada a partir da divulgação do endereço eletrônico do questionário, nas redes sociais de um dos projetos de extensão da Universidade Federal da Paraíba (UFPB) que divulga informações sobre segurança de alimentos. O questionário ficou disponível no período de 01/10/2020 a 03/10/2020.

Para a análise estatística foi utilizada estatística descritiva para exposição dos resultados em percentuais de frequência e foi realizada estatística inferencial por meio do teste de Qui-Quadrado de Pearson (Plackett, 1983) com nível de significância de 5\% para verificar a homogeneidade dos meios de comunicação utilizados para pesquisas sobre higiene, bem como a associação entre as variáveis de higiene pessoal ou higiene de alimentos e os meios de comunicação utilizados para a busca de informação. O programa R foi utilizado para realização dos testes (R Core Team, 2020).

\section{Resultados e Discussão}

Dos 201 questionários, apenas 198 dos respondentes foram computados na análise de dados, pois três questionários não foram totalmente respondidos.

Dentre os respondentes incluídos na pesquisa, 78\% são do gênero feminino, e 22\% são do gênero masculino. Quanto a faixa etária, $46 \%$ estavam entre 18 e 29 anos de idade, 39\% tinham entre 30 a 44 anos, 12\% e 3\% estavam, respectivamente, com 45 a 60 e acima de 60 anos (Figura 1). 
Figura 1. Histograma da frequência em percentuais de respondentes classificados por gênero e faixa etária.

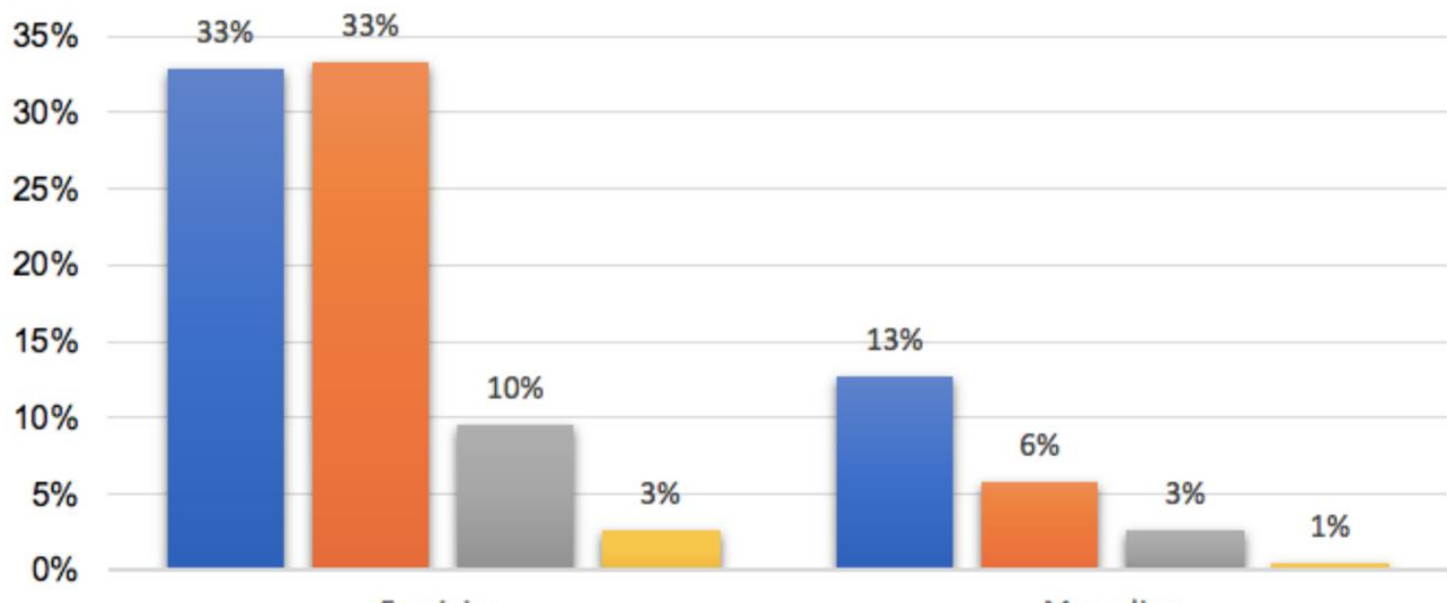

Feminino

Masculino

18 a 29 anos 30 a 44 anos 45 a 60 anos Acima de 60 anos

Fonte: Autores (2020).

Quanto a escolaridade, $45 \%$ e 34\% possuem ensino superior e pós-graduação, respectivamente. 13\% dos entrevistados atuavam profissionalmente como manipuladores de alimentos e, dentre eles, $92 \%$ buscou informações sobre as novas medidas de higiene para prevenir a contaminação pelo vírus no ambiente de trabalho (Tabela 1).

Tabela 1. Caraterização sociodemográfica dos participantes.

\begin{tabular}{|c|c|c|c|c|}
\hline Variáveis & \multicolumn{4}{|c|}{ Respostas } \\
\hline \multirow{2}{*}{ Gênero } & Feminino & \multicolumn{2}{|c|}{ Masculino } & Não declarou \\
\hline & $78 \%$ & \multicolumn{2}{|c|}{$22 \%$} & $0 \%$ \\
\hline \multirow[b]{2}{*}{ Faixas etárias } & 18 a 29 anos & 30 a 44 anos & 45 a 60 anos & Acima de 60 \\
\hline & $46 \%$ & $39 \%$ & $12 \%$ & $3 \%$ \\
\hline \multirow{2}{*}{ Escolaridade } & Ensino fundamental & Ensino médio & Ensino superior & Pós-graduação \\
\hline & $2 \%$ & $20 \%$ & $45 \%$ & $34 \%$ \\
\hline Atua como & \multicolumn{2}{|c|}{ Sim } & \multicolumn{2}{|c|}{ Não } \\
\hline $\begin{array}{l}\text { manipulador de } \\
\text { alimentos }\end{array}$ & \multicolumn{2}{|c|}{$13 \%$} & \multicolumn{2}{|c|}{$87 \%$} \\
\hline
\end{tabular}

Fonte: Autores (2020).

Sabe-se que os serviços de alimentação são considerados locais propícios a transmissão de doenças de origem alimentar, devido a manipulação inadequada e falta de higiene (Young et al., 2020). E que a qualificação quanto as boas práticas de higiene é a estratégia mais eficaz para melhorar a segurança alimentar (Clark et al., 2017). Por este motivo, é exigido certificação de curso de boas práticas de fabricação de alimentos para os manipuladores de alimentos em locais de produção coletiva de alimentação, no Brasil e em muitos países (Brown et al., 2014; Clark et al., 2017; Young et al., 2020). Portanto, o 
interesse destes profissionais em buscar informações sobre práticas de prevenção a contaminação na produção de alimentos é um aspecto positivo para este momento de pandemia.

A maioria dos respondentes residiam no estado da Paraíba. Mas, houve participação significativa de outros estados, como Ceará, Pernambuco e São Paulo (Figura 2).

Figura 2. Mapa da localização dos respondentes da pesquisa.

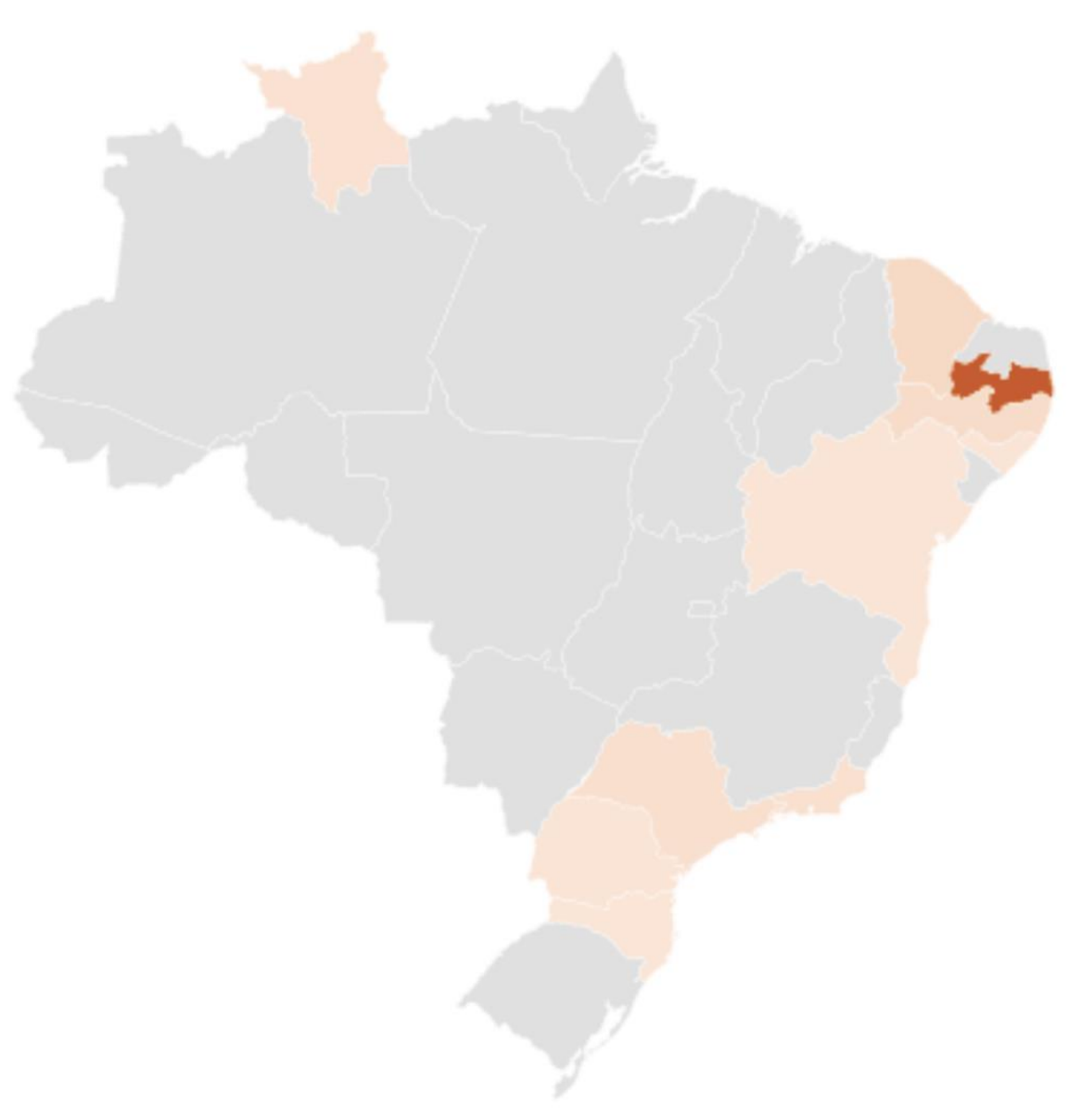

\section{Série1}

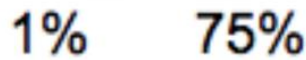

Fonte: Autores (2020).

Em março, quando iniciaram as medidas para minimizar a transmissão da COVID-19 no Brasil (Ministério da Saúde, 2020), muitas ações em paralelo também foram iniciadas visando reduzir os danos colaterais econômicos, sociais, emocionais e de saúde que seriam resultantes da restrição do funcionamento de escolas, universidades, locais de convívio comunitário, transporte público, além de outros locais onde há aglomeração de pessoas, como eventos sociais, esportivos, teatros, cinemas e estabelecimentos comerciais (Vargas, 2020; Neeltje Van Doremalen, 2020; ANVISA, 2020).

Universidades desenvolveram diversas ações de pesquisa e extensão para o combate aos efeitos da pandemia (Ferreira, 2020). Dentre as quais estão as ações de extensão voltadas a divulgação de informações sobre higiene (UNIFESSPA, 2020). Tendo em vista que a coleta de dados ocorreu por meio das redes sociais de um projeto de extensão universitária, estes dados 
podem representar a capacidade de alcance de ações educativas divulgadas gratuitamente e promovidas por instituições de ensino, pesquisa e extensão.

A extensão é uma ferramenta para o compartilhamento de saberes entre a universidade e a sociedade, sendo uma oportunidade de troca para aprimorar o processo educativo, cultural e científico que articula o ensino e a pesquisa de forma indissociável e viabiliza a relação transformadora entre a universidade e a sociedade (Solto, 2016).

Os dados deste estudo (Tabela 2) revelaram que 95\% dos participantes buscaram informações sobre higiene para mitigar a contaminação do coronavírus durante o período de distanciamento social decorrente da pandemia, sobretudo no início desse período. Dentre esses, $86 \%$ declararam que buscaram informações específicas sobre higiene de alimentos para esta finalidade.

Tabela 2. Fontes de informação sobre higiene durante o período de isolamento social em decorrência da pandemia do novo coronavírus.

\begin{tabular}{|c|c|c|c|c|c|}
\hline \multirow{2}{*}{$\begin{array}{l}\text { 1. Buscou informações sobre higiene para mitigar a } \\
\text { contaminação do coronavírus durante o período de } \\
\text { distanciamento social decorrente da pandemia do } \\
\text { novo coronavírus? }\end{array}$} & \multicolumn{3}{|c|}{ Sim } & \multicolumn{2}{|l|}{ Não } \\
\hline & \multicolumn{2}{|r|}{$95 \%$} & & \multicolumn{2}{|l|}{$5 \%$} \\
\hline \multirow{2}{*}{$\begin{array}{l}\text { 2. Buscou informações específicas sobre higiene de } \\
\text { alimentos para mitigar a contaminação do } \\
\text { coronavírus durante o período de distanciamento } \\
\text { social decorrente da pandemia do novo } \\
\text { coronavírus? }\end{array}$} & \multicolumn{2}{|r|}{ Sim } & & \multicolumn{2}{|l|}{ Não } \\
\hline & & $86 \%$ & & $14 \%$ & \\
\hline \multirow{2}{*}{$\begin{array}{l}\text { 3. Qual(s) meio(s) de informação utilizou para obter } \\
\text { informações sobre como mitigar a contaminação do } \\
\text { coronavírus durante o período de distanciamento } \\
\text { social decorrente da pandemia do novo } \\
\text { coronavírus? }\end{array}$} & Internet & Telejornais & E-book & $\begin{array}{l}\text { Livros e } \\
\text { jornais } \\
\text { impressos }\end{array}$ & $\begin{array}{c}\text { Valor } \\
\mathrm{X}^{2 *}\end{array}$ \\
\hline & $78 \%$ & $11 \%$ & $30 \%$ & $5 \%$ & 14,81 \\
\hline
\end{tabular}

*Valor qui-quadrado para homogeneidade. Fonte: Autores (2020).

Um dos setores que sofreu grande impacto durante este período foi o setor de alimentação fora do lar que, mesmo após o processo de reabertura, manteve-se em baixa devido as passiveis mudanças no comportamento do consumidor, que foram motivadas, em parte, por sentimentos de medo em relação ao vírus (Baker et al, 2020).

Como resultado, pode-se destacar a queda acentuada nos gastos com restaurantes, varejo, viagens aéreas e transporte público revelada em outros estudos, que também evidenciaram um aumento significativo nos gastos com entrega de alimentos, consistente com as famílias substituindo as refeições em restaurantes por refeições em casa (The food industry association, 2020; Grashuis, 2020, Romeo-Arroyo, 2020).

Esse processo tem elevado, por outro lado, a busca de informações sobre técnicas de preparo de alimentos, cuidados de higiene e outros aspectos para o preparo seguro de alimentos em ambiente domésticos (Ministério da Saúde, 2016; Baker et al, 2020; Rodrigues et al, 2020).

Nesse contexto foi observado que $78 \%$ dos participantes indicaram buscar informações por meio da internet, $37 \%$ buscaram em telejornais, 30\% utilizaram o manual de Boas Práticas de prevenção ao coronavírus em Serviços de Alimentação, um e-book produzido pelo Departamento de Gastronomia da UFPB (Rodrigues et al, 2020). E, apenas 5\% do público buscaram informações em livros e em jornais impressos (Figura 3). 
Houve diferença significativa entre os tipos de fonte de informação, ou seja, a utilização de internet, seguida por telejornais e o e-book sobre boas práticas de prevenção ao Coronavírus foram às estratégias mais utilizadas para obter informação sobre práticas de higiene no grupo avaliado.

Figura 3. Fontes de informação sobre higiene de alimentos utilizadas pelos participantes.

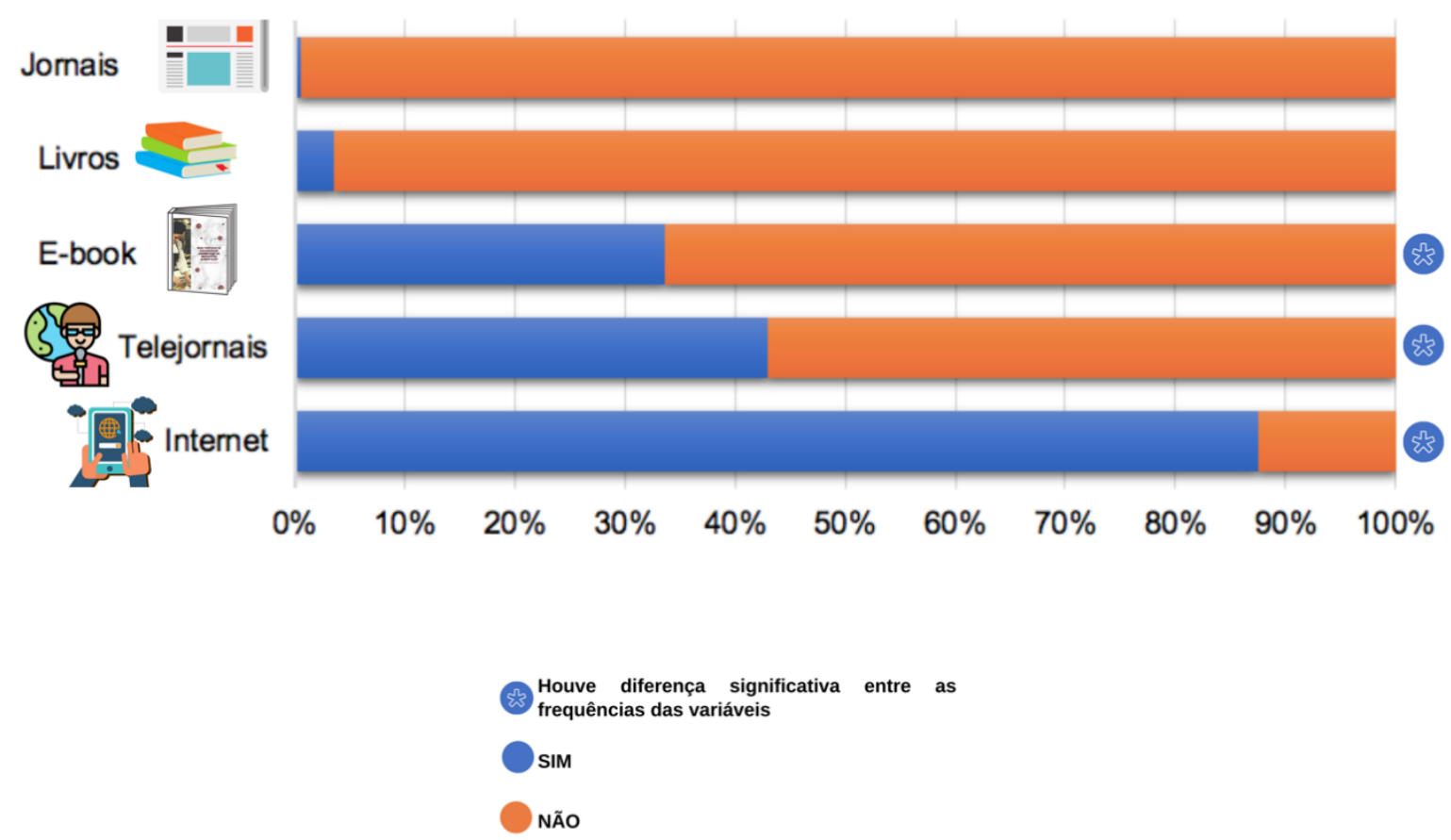

Fonte: Autores (2020).

Um estudo publicado pela Universidade Britânica de Columbia descreveu os principais temas de buscas pesquisados na internet durante a pandemia do novo coronavírus em 268 países, e divulgou dados quantitativos quanto aos temas pesquisados. A busca por informações sobre "alimentos" está entre as 30 hashtags mais utilizadas no mundo no período de distanciamento social nestes países e a língua portuguesa é a segunda mais utilizada para busca de informações (Abdul-Mageed, 2020). Essas características evidenciam a necessidade de oferecer informações corretas e de fontes seguras sobre o assunto.

A crise decorrente da COVID-19 também acelerou a mudança para serviços digitais e a digitalização tornou-se uma necessidade básica (Schilirò, 2020). Ou seja, as tecnologias digitais passaram a desempenhar um papel crucial na manutenção da vida quotidiana, bem como das atividades econômicas e sociais (World Economic Forum, 2020). Com isso, a pandemia do coronavírus pode se tornar um ponto de inflexão para a digitalização ao acelerar a maturidade da tecnologia digital (Schilirò, 2020). Sobretudo no que diz respeito ao mercado de alimentos e estratégias de qualificação para o cuidado com a manipulação.

Também foi questionado aos participantes quais hábitos de higiene mudaram após a instalação das medidas comunitárias para mitigação da doença (Tabela 3). E quais as mudanças que ocorreram nas práticas de higiene durante a produção de alimentos (Tabela 4). 
Research, Society and Development, v. 10, n. 1, e30910111739, 2021

(CC BY 4.0) | ISSN 2525-3409 | DOI: http://dx.doi.org/10.33448/rsd-v10i1.11739

Tabela 3. Mudanças de hábitos de higiene pessoal e associação com as fontes de informação sobre higiene de alimentos.

Valor-p do teste qui-quadrado para qualidade de ajuste com

1. Aumentou a frequência e o tempo de duração de lavagem das mãos após o início da pandemia do novo coronavírus?

Sim

Não

Todas as
fontes E-book Internet

$\begin{array}{lllll}77 \% & 9 \% & 0,02 & >0,05 & >0,05\end{array}$

\begin{tabular}{|c|c|c|c|c|c|}
\hline \multirow{2}{*}{$\begin{array}{l}\text { 2. Passou a executar o processo correto de } \\
\text { higienização das mãos após o início da } \\
\text { pandemia do novo coronavírus? }\end{array}$} & Sim & \multicolumn{4}{|l|}{ Não } \\
\hline & $40 \%$ & $46 \%$ & $>0,05$ & 0,01 & $>0,05$ \\
\hline \multirow{2}{*}{$\begin{array}{l}\text { 3. Passou a utilizar máscaras corretamente após } \\
\text { o início da pandemia do novo coronavírus? }\end{array}$} & Sim & Não & & & \\
\hline & $48 \%$ & $47 \%$ & 0,01 & $>0,05$ & $>0,05$ \\
\hline \multirow{2}{*}{$\begin{array}{l}\text { 4. Aumentou a frequência de higienização do } \\
\text { celular após o início da pandemia do novo } \\
\text { coronavírus? }\end{array}$} & Sim & Não & & & \\
\hline & $65 \%$ & $21 \%$ & $>0,05$ & 0,01 & 0,00 \\
\hline
\end{tabular}

Fonte: Autores (2020). 
Tabela 4. Mudanças de hábitos de higiene de alimentos e associação com as fontes de informação sobre higiene de alimentos.

Valor-p do teste qui-quadrado para qualidade de ajuste com

1. Passou a higienizar os vegetais corretamente após o início da pandemia do novo coronavírus?
Sim

Não

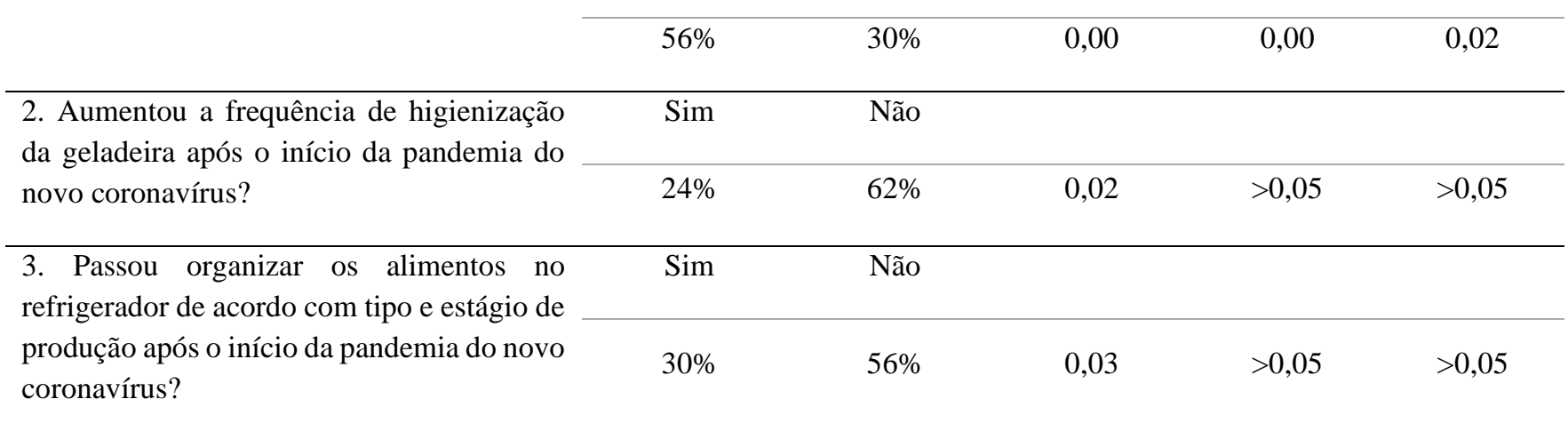

\begin{tabular}{|c|c|c|c|c|c|}
\hline 4. Aumentou a frequência de higienização & Sim & Não & & & \\
\hline $\begin{array}{l}\text { alimentos após o início da pandemia do } \\
\text { novo coronavírus? }\end{array}$ & $31 \%$ & $55 \%$ & 0,01 & $>0,05$ & $>0,05$ \\
\hline 5. Passou a descongelar carnes sob & Sim & Não & & & \\
\hline pandemia do novo coronavírus? & $22 \%$ & $64 \%$ & $>0,05$ & $>0,05$ & $>0,05$ \\
\hline 6. Não permite que os alimentos prontos & Sim & Não & & & \\
\hline ambiente? & $16 \%$ & $70 \%$ & $>0,05$ & $>0,05$ & $>0,05$ \\
\hline 7. Passou a verificar a validade dos & Sim & Não & & & \\
\hline da pandemia do novo coronavírus? & $56 \%$ & $30 \%$ & 0,00 & 0,01 & $>0,05$ \\
\hline 8. Passou a higienizar as embalagens de & Sim & Não & & & \\
\hline pandemia do novo coronavírus? & $71 \%$ & $15 \%$ & 0,00 & $>0,05$ & $>0,05$ \\
\hline 9. Passou a usar máscara ao receber & Sim & Não & & & \\
\hline pandemia do novo coronavírus? & $65 \%$ & $21 \%$ & 0,00 & $>0,05$ & 0,01 \\
\hline 10. Passou a descartar as embalagens de & Sim & Não & & & \\
\hline coronavírus? & $47 \%$ & $38 \%$ & 0,01 & $>0,05$ & 0,02 \\
\hline
\end{tabular}

Fonte: Autores (2020).

Dentre os participantes que buscaram informações sobre higiene, 77\% afirmaram que aumentaram a frequência de lavagem das mãos com tempo de duração superior a vinte segundos. Também foi observada uma associação positiva desta mudança de hábito com a busca de informações sobre higiene em qualquer que seja a fonte de busca (Figura 4). O uso de soluções 
sanitizantes após a lavagem das mãos também foi uma mudança de hábito para $40 \%$ dos participantes e esta prática teve uma associação positiva com o acesso ao e-book produzido pela UFPB.

Com relação ao uso de máscaras, $48 \%$ dos respondentes declararam que passaram a usa-la corretamente após obter informações sobre higiene de alimentos e esta mudança teve uma associação positiva com qualquer que seja a fonte de informação. Também foi contatado que $65 \%$ dos participantes aumentaram a frequência de higienização do celular após o início da pandemia, e esta mudança teve uma associação positiva, ou seja, foi fortemente influenciada pelas informações divulgadas no e-book e na internet.

Figura 4. Mudanças de hábitos de higiene e associação com as fontes de informação sobre higiene de alimentos.

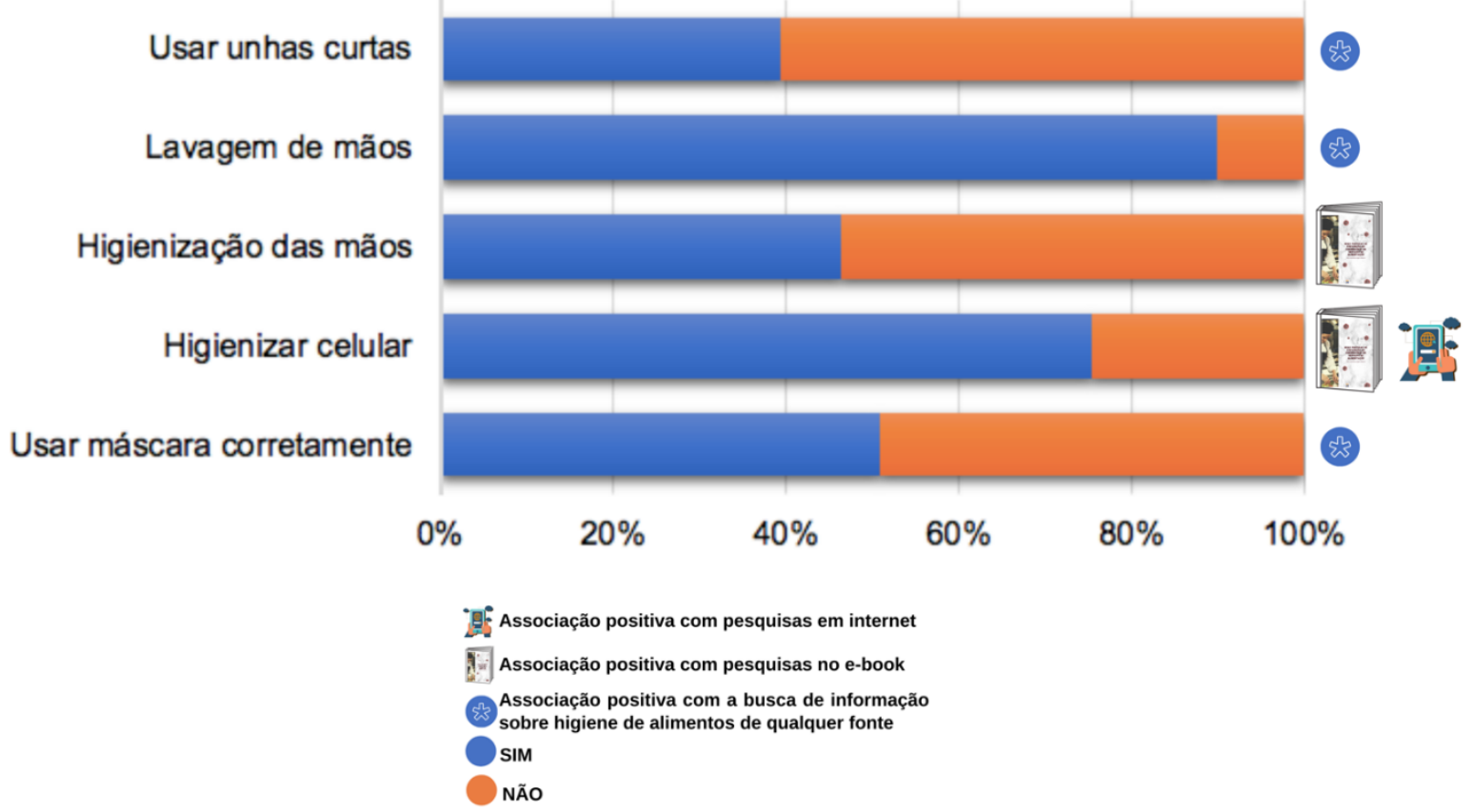

Fonte: Autores (2020).

Quanto às mudanças de hábitos relacionadas à higiene de alimentos, 56\% declararam que passaram a higienizar os vegetais com solução clorada ao recebê-los em sua residência ou estabelecimento, $24 \%$ aumentaram a frequência de higienização da geladeira e 30\% passaram a organizar os alimentos no refrigerador separados por grupos quanto ao estágio de produção e o tipo de alimento, evitando contaminação cruzada. Ainda, $31 \%$ dos respondentes aumentaram a frequência de limpeza do ambiente de produção, evitando contaminação cruzada e mantendo a organização.

O processo de controle de temperatura de alimentos considerados de alto risco para contaminação obteve uma melhora de $22 \%$ para o descongelamento das carnes em temperaturas fora da zona considerada de risco (entre $5^{\circ}$ e $60^{\circ} \mathrm{C}$ ). E $16 \%$ não deixam mais os alimentos prontos por tempo superior a duas horas em temperatura ambiente, o que favorece a proliferação de microrganismos patogênicos.

Ainda foi observado que $56 \%$ e $71 \%$ dos participantes, respectivamente, mudaram os hábitos de aquisição de alimentos no que diz respeito a observar o prazo de validade e higienização das embalagens ao recebê-los.

Os cuidados com alimentos entregues no domicilio também foram evidenciados com $65 \%$ dos participantes declarando utilizar máscara para o recebimento de delivery e 47\% passando a descartar embalagens de papelão. 
A Figura 5 destaca a relação de influência da mudança destes hábitos nas práticas de higiene de alimentos com a obtenção de conhecimento neste período de pandemia e revela que houve uma associação positiva entre as mudanças hábitos e o conhecimento compartilhado de qualquer que seja a fonte.

A internet influenciou positivamente nos hábitos de recebimento de alimentos prontos como o uso de máscara para o recebimento de delivery e o descarte das embalagens de papelão. O uso do e-book da UFPB teve uma influência nas práticas de aquisição e recebimento de alimentos como controle dos aspectos de validade e higienização de vegetais.

Figura 5. Mudanças de hábitos de higiene de alimentos e associação com as fontes de informação sobre higiene de alimentos

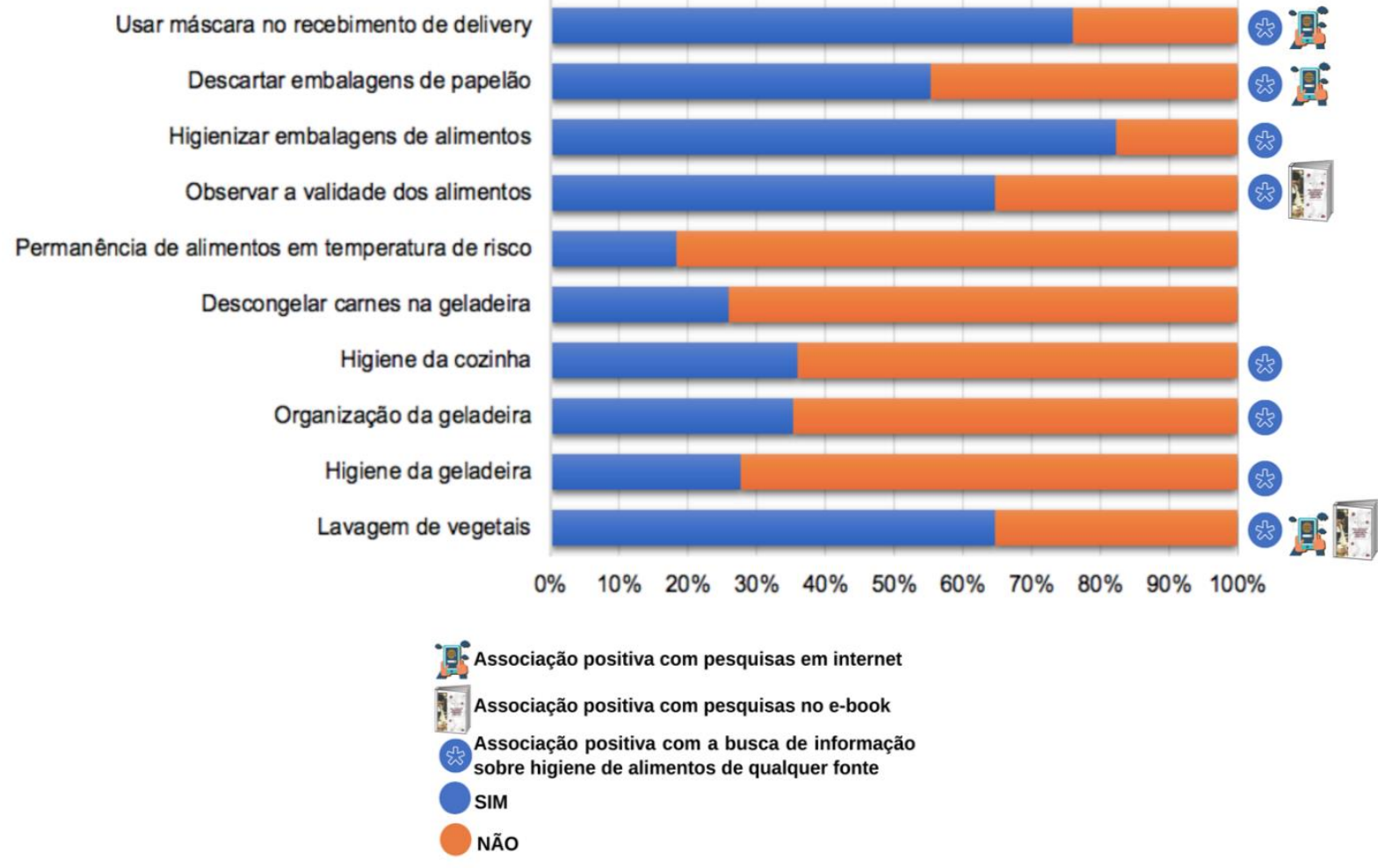

Fonte: Autores (2020).

Em uma revisão sistemática que avaliou o efeito da educação em segurança de alimentos e intervenções de treinamentos foi observado (total de 85 estudos) que houve melhorias em todos os aspectos observados, e em todas as etapas de produção de alimentos (Young et al., 2020).

As Boas Práticas de Fabricação quando implantadas e bem implementadas nas áreas de produção de alimentos podem prevenir de forma eficaz a propagação de qualquer contaminação biológica por meio da manipulação inadequada, incluindo a contaminação por vírus (ANIVSA, 2020; Rodrigues et al., 2020; WHO, 2020).

Importante destacar que dentre os aspectos incluídos nas práticas de higiene para prevenção da Covid-19 na produção de alimentos para coletividades estão o distanciamento de pelo menos um metro nas áreas de produção e o uso de máscaras (Rodrigues et al, 2020; WHO, 2020).

Contudo, apesar dos alimentos não transmitirem o SARS-CoV-2, as superfícies de utensílios, equipamentos e embalagens ou de ambientes de produção e consumo de alimentos podem conter o vírus se não forem bem higienizadas (Wu et al, 2020; Morawska \& Milton, 2020). Isso porque, já foi relatada a permanecer do vírus ativo por até 72 horas em algumas destas 
superfícies, conotando assim a importância de se manter as práticas de higiene adequadas tanto em ambientes de produção coletivas, como no ambiente doméstico (Rodrigues et al, 2020; WHO, 2020).

\section{Conclusão}

É possível concluir que o período de pandemia gerou mudanças importantes nos hábitos de higiene da população estudada. E que estratégias de educação sanitária através de meios de comunicação podem gerar transformações positivas nas práticas de produção de alimentos seguros. Isso estimula a produção e divulgação de materiais educativos com ampla exposição, pois esses podem contribuir com as boas práticas de fabricação de alimentos.

Também se faz necessário à realização de novos estudos acerca desta temática, principalmente após a flexibilização das medidas de mitigação para Covid-19, visando manter os cuidados que garantam a seguridade dos alimentos.

\section{Referências}

Abdul-Mageed, M., Elmadany, A., Pabbi, D., Verma, K., \& Lin, R., (2020). Mega-COV: A Billion-Scale Dataset of 100+ Languages for COVID-19. http://arxiv.org/abs/2005.06012

ANVISA. Agência Nacional de Vigilância Sanitária. (2020). Orientações para os serviços de alimentação com atendimento direto ao cliente durante a pandemia de Covid-19. http://www.vigilanciasanitaria.sc.gov.br/index.php/149-noticias/noticias-2020/1148-anvisa-esclarece-sobre-o-novo-coronavirus-e-os-alimentos.

Baker, S. R., Farrokhnia, R. A., Meyer, S., Pagel, M., \& Yannelis, C. (2020). How Does Household Spending Respond to an Epidemic? Consumption during the 2020 COVID-19 Pandemic. Rev Asset Pricing Stud. 1(26949). 1-29. 10.3386 / w26949.

Brown, L. G., Le, B., Wong, M. R., Reimann, D., Nicholas, D., Faw, B., et al. (2014). Restaurant manager and worker food safety certification and knowledge. Foodborne Pathogens and Disease. 11(11). 1-9. doi: 10.1089/fpd.2014.1787.

Clark, J., Crandall, P. G., \& O’Bryan, C. (2017). Climbing the Intervention Ladder to handwashing compliance: A review and directions for future research. Food Control. 84(1). 544-51. https://doi.org/10.1016/j.foodcont.2017.09.009.

Ferreira, I. (2020). Ações de universidades públicas evidenciam importância da ciência no combate à covid. https://jornal.usp.br/ciencias/acoes-de-universidadespublicas-evidenciam-importancia-da-ciencia-no-combate-a-covid/.

Grashuis, J., Skevas, T., Segovia, M. S. (2020). Grocery shopping preferences during the COVID-19 pandemic. Sustainability. 12(5369). 2-10. $10.3390 / \mathrm{su} 12135369$.

Li, Y., Qian, H., Hang, J., Chen, X., Hong, L., Liang, P., et al. (2020). Evidence for probable aerosol transmission of SARS-CoV-2 in a poorly ventilated restaurant. 1-19. https://doi.org/10.1101/2020.04.16.20067728.

Ministério da Saúde (MS). (2013) Conselho Nacional de Saúde. Resolução nº 466, de 12 de dezembro de 2012. Diário Oficial da União. https://bvsms.saude.gov.br/bvs/saudelegis/cns/2013/res0466_12_12_2012.html.

Ministério da Saúde (MS). Conselho Nacional de Saúde. Resolução $\mathrm{n}^{\mathrm{o}}$ 510, de 7 de abril de 2016. Diário Oficial da União 2016. https://bvsms.saude.gov.br/bvs/saudelegis/cns/2016/res0510_07_04_2016.html.

Ministério da Saúde (MS). (2020). Ministério da Saúde declara transmissão comunitária nacional. https://www.saude.gov.br/noticias/agencia-saude/ 46568ministerio-da-saude-declara-transmissao-comunitaria-nacional.

Ministério da Saúde (MS). (2020). PORTARIA no 432, de 19 de março de 2020. Diário oficial da União. http://www.in.gov.br/en/web/dou/-/portaria-n-454-de20-de-marco-de-2020-249091587.

Morawska, L. \& Milton, D. K. (2020). It Is Time to Address Airborne Transmission of Coronavirus Disease 2019 (COVID-19). Clin Infect Dis. 71(1). 23112313. https://doi.org/10.1093/cid/ciaa939.

Neeltje van Doremalen, P. (2020). Infectious (National Institute of Allergy and, Diseases), Trenton Bushmaker BSNI of A and, Diseases), Dylan H. Morris MP (Princeton U. Aerosol and Surface Stability of SARS-CoV-2 as Compared with SARS-CoV-1). N Engl J Med, 0-2.

Pereira, A. S., Shitsuka, D. M., Parreira, F. J., Shitsuka, R. (2018). Metodologia da pesquisa científica. Ed. UAB/NTE/UFSM. https://repositorio.ufsm.br/bitstream/handle/1/15824/Lic_Computacao_Metodologia-Pesquisa-Cientifica.pdf?sequence=1

Plackett, R. L. (1983). Karl Pearson and the Chi-Squared Test. Int Stat Rev / Rev Int Stat. 51(1). 59-72. https://doi.org/10.2307/1402731.

R Core Team (2020). R: A language and environment for statistical. R Foundation for Statistical Computing, Vienna, Austria.

Rodrigues, N. P. A., Silva, D. R. da., Júnior, A. G., \& Souza, E. L. (2020) Boas Práticas de Prevenção ao Coronavírus em Serviços de Alimentação: Com tradução para Inglês e Francês. https://drive.google.com/drive/u/0/folders/1AIGmgrFlYO8yv3ABEl V8wrAU 1SX0hkXr. 
Research, Society and Development, v. 10, n. 1, e30910111739, 2021 (CC BY 4.0) | ISSN 2525-3409 | DOI: http://dx.doi.org/10.33448/rsd-v10i1.11739

Rodriguez-Morales, A. J., Gallego, V., Escalera-Antezana, J. P., Méndez, C. A., Zambrano, L. I., Franco-Paredes, C., et al. (2020). COVID-19 in Latin America: The implications of the first confirmed case in Brazil. Travel Med Infect Dis. 35(101613). 1-3. 10.1016/j.tmaid.2020.101613.

Romeo-Arroyo, E., Mora, M., Vázquez-Araújo, L. (2020). Consumer behavior in confinement times: Food choice and cooking attitudes in Spain. International Journal of Gastronomy and Food Science. 21(100226). 1-5. https://doi.org/10.1016/j.ijgfs.2020.100226

Schilirò, D., (2020). Towards digital globalization and the covid-19 challenge Towards digital globalization and the covid-19 challenge. Munich Personal RePEc Archive. 1(100504). 1-8. https://mpra.ub.uni-muenchen.de/100504/.

Souto, M. R. M. (2006). Conceitos De Extensão Universitária. https://www.ets.ufpb.br/pdf/2013/1\%20Universidade\%20e\%20Sociedad e/US\%2013_Texto\%201\%20Serrano_Conceitos\%20de\%20extensao\%20universitaria.pdf.

Teixeira, L. A. \& de Carvalho, W. R. G. (2020). SARS-CoV-2 em superfícies: persistência e medidas preventivas - uma revisão sistemática. Journal Health NPEPS, 5(2) 1-16. http://dx.doi.org/10.30681/252610104873.

The food industry association. (2020). U.S. Grocery Shopper Trends: The Impact of COVID-19. https://www.fmi.org/blog/view/webinar-recordingspublic/2020/05/07/u.s -grocery-shopper-trends-the-impact-of-covid-19.

UNIFESSPA. (2020). I Congresso Andifes destaca ações das universidades federais no combate ao coronavírus. https://www.unifesspa.edu.br/noticias/4644-icongresso-andifes-destaca-acoes-das-universidades-federais-no-combate-ao-coronavirus.

Vargas, J. R. N. The COVID-19 pandemic. (2020). Revista de la Facultad de Medicina. 68(1). 7-8. https://doi.org/10.15446/revfacmed.v68n1.86482.

Villapol, S. Gastrointestinal symptoms associated with COVID-19: impact on the gut microbiome. Transl Res. (2020) 226 (1), 57-69. https://doi.org/10.1016/j.trsl.2020.08.004.

WHO. World Health Organization. (2020). Coronavirus disease (COVID-19): How is it transmitted?. Q\&A Detail. 2020. Coronavirus disease (COVID-19) pandemic. https://www.who.int/news-room/q-a-detail/q-a-how-is-covid-19-transmitted.

WHO. World Health Organization. (2020). COVID-19 and food safety: guidance for food businesses. Interim Guid. https://apps.who.int/iris/rest/bitstreams/1274400/retrieve.

World Economic Forum. (2020). These charts show how COVID-19 has changed consumer spending around the world. https://www.weforum.org/agenda/ 2020/05/ coronavirus-covid19-consumers-shopping-goods-economics-industry/.

Wu D., Wu T., Liu, Q. \& Yang, Z. (2020). The SARS-CoV-2 outbreak: What we know. Int J Infect Dis. 94(1), 44-8. https://doi.org/10.1016/j.ijid.2020.03.004.

Young, I., Waddell, L. A., Wilhelm, B. J., Greig, J. (2020). A systematic review and meta-regression of single group, pre-post studies evaluating food safety education and training interventions for food handlers. Food Research International. 128(108711). 1-8. https://doi.org/10.1016/j.foodres.2019.108711. 